\title{
Internacionalización de la educación superior en Costa Rica La experiencia de una maestría de doble titulación de la Universidad Nacional
}

\author{
Internationalization of Higher Education in Costa Rica \\ The experience of a dual Master's degree at Universidad Nacional
}

\author{
Arlette Pichardo Muñiz' \\ Universidad Nacional, Costa Rica. arlette.pichardo.muniz@una.cr
}

Fecha de recepción: 20/08/2014. Reenvios: 29/9/2014 y 20/8/2015. Fecha de aceptación: 28/01/2016. Fecha de publicación: 13/06/2016.

\begin{abstract}
Resumen: Costa Rica ha propiciado la movilidad internacional estudiantil y docente desde hace varias décadas, incluso como sede de programas internacionales de educación superior. El Centro Internacional de Política Económica para el Desarrollo Sostenible (CINPE) de la Universidad Nacional (UNA) en la actualidad imparte la Maestría en Gerencia del Comercio Internacional (MGCl), en doble titulación con el Instituto Tecnológico de Santo Domingo (INTEC), República Dominicana. Esta es la primera de su género en el país -según se conoce hasta la fecha- $y$, quizás, en Centroamérica, donde una universidad costarricense aporta el currículo y asume el liderazgo académico, incorporando la transculturalidad como valor agregado. El objetivo del artículo es difundir lecciones de esta experiencia que puedan ser útiles para otras iniciativas. En términos generales, los resultados de la sistematización indican que: (1) la inclusión de las dimensiones internacional e intercultural supone, primero, la internacionalización en casa; (2) la creación de condiciones iniciales para escalar peldaños superiores en el proceso de internacionalización, no ocurre de la noche a la mañana; (3) la gestión y gerencia suponen soluciones innovadoras y consensuadas; y (4) hace falta poner la debida atención en la armonización de procedimientos, instrumentos y formularios. Sobre la base de los principales hallazgos se presentan algunas conclusiones, en particular para la discusión de las implicaciones de un programa de esta naturaleza u otros con características similares.
\end{abstract}

Palabras clave: Costa Rica, educación superior, internacionalización, maestría de doble titulación, República Dominicana.

\begin{abstract}
Costa Rica has promoted international student and academic mobility for several decades, going as far as hosting international higher education programs. The International Center on Economic Policy for Sustainable Development (CINPE, for its acronym in Spanish) at Universidad Nacional (UNA, for its acronym in Spanish) has
\end{abstract}

$1 \quad$ La autora agradece los comentarios de la Dra. Alba Henríquez, coordinadora nacional de la MGCI del CINPE-UNA en doble titulación con el INTEC, del Ph.D. Rafael Díaz Porras, académico del CINPE y del Dr. Frank Dellmann, académico de la Universidad Münster y profesor visitante del CINPE y la corrección de estilo de la M.Sc. Hazel Vargas. Por supuesto, que tanto la versión final como las apreciaciones contenidas en ella son de su absoluta responsabilidad y, no necesariamente, representan o comprometen a la institución de la que forma parte. 
joined the Technology Institute of Santo Domingo (INTEC, for its acronym in Spanish), Dominican Republic, to teach the Master's Degree in International Trade Management. This is the first program of its kind known to date in the country and probably in Central America, in which a Costa Rican university provides the curriculum and takes a leading academic position, with transculturality becoming an added value. This paper is aimed at sharing the lessons learned in this experience that may be useful in similar initiatives. In general, the results of the systematization suggest the following: (1) including international and intercultural perspectives implies internationalizing locally; (2) creating initial conditions to advance in the internationalization process does not happen overnight; (3) management implies innovative solutions agreed by all; and (4) due attention should be paid to the harmonization of procedures, instruments, and forms. Some conclusions are drawn based on the main findings, particularly regarding the implications of a program of this nature or others with similar characteristics.

Keywords: Costa Rica, Higher Education, internacionalization, Dual Master's degree, Dominican Republic.

\section{Introducción}

Desde hace ya varias décadas Costa Rica ha venido participando en el concierto de la internacionalización de la educación superior, ya sea por medio de la articulación de redes y organizaciones académicas regionales, la instauración de instituciones internacionales de educación superior, la creación de programas de movilidad estudiantil por parte de algunas universidades o el incentivo al turismo educativo.

La internacionalización de la educación superior resulta inherente al origen mismo de la universidad, del latín universitas, -ätis que alude a la cualidad de universal. Desde que se conoce de su existencia, la universidad tiende a abrir sus puertas a estudiantes de distinto lugar de origen. De modo que -por definición- trascender, en su proyección, las barreras del espacio geográfico donde se sitúa, resulta consustancial a la esencia y valores del quehacer universitario.

En los últimos años, las experiencias sobre este particular están en proceso de crecimiento y abundancia, aunque se puede decir que existe poco conocimiento divulgado acerca de su dinámica actual, y su incursión en las esferas de la política pública aparece en forma tardía (Chadee y Naidoo, 2009).

Knight (1994, 2003, 2004, 2006 y 2010), desde sus trabajos iniciales sobre el tema, insiste en que se trata de un término con múltiples significados. Al efecto, indica que en algunas universidades la internacionalización puede restringirse a intercambio académico: movilidad estudiantil, docente y de investigación, cursos de idiomas, vínculos y redes, asociaciones y consorcios 
(partnership $)^{2}$. Mientras que, en otras, se le entiende como la transmisión de educación a otros países, por medio de sucursales y filiales universitarias, acuerdos o convenios de franquicias, cara a cara (face to face) o por medios a distancia (virtuales) ${ }^{3}$. Señala también que, para la gran mayoría de universidades, consiste en la inclusión de las dimensiones internacional e intercultural en los diversos ámbitos del quehacer universitario ${ }^{4}$.

Tal conceptualización general, que no excluye consideraciones más específicas, ha logrado un amplio consenso, quizás por su simpleza; y permea la conceptualización más generalizada y difundida, expandiéndose prácticamente en forma universal en diversos ámbitos del quehacer universitario (curricula, proceso de enseñanza, programas de desarrollo y diseño de políticas, misión institucional, funciones globales universitarias y otros) $)^{5}$.

En la bibliografía sobre internacionalización de la educación superior en América Latina y el Caribe se destacan algunos esfuerzos, aunque su aplicación a casos prácticos es más escasa. Las diversas aproximaciones van desde la vinculación con funciones sustantivas y la redefinición de la misión institucional de las universidades (Gacel y Ávila, 2008); su conceptualización como paradigma para la ciudadanía global (Gacel-Ávila, 2003); desafíos y tareas (Ramírez Sánchez,

2 La internacionalización empieza a formar parte de las estrategias de universidades europeas y norteamericanas en particular a partir del fomento e incentivo de la movilidad académica, desplazando profesorado a países del sur para impartir docencia, especialmente en verano y recesos institucionales. En su momento, el Programa Erasmus Mundus, mediados de 1980, con financiamiento de la Unión Europea, fue considerado líder en cooperación internacional para la educación superior, por medio de diferentes programas, incluida la doble titulación. En la práctica transformó la dimensión internacional de las universidades europeas y creó condiciones para las siguientes etapas de internacionalización. En América Latina y el Caribe, razones de costo, normativas nacionales, tiempos de estudio y calendarios de trabajo, heterogeneidad en los criterios de reconocimiento o convalidación, homologación y equiparación de estudios y títulos, y la necesidad de mayores requirimientos de calidad impidieron una mayor y mejor apropiación de sus beneficios.

La Global Alliance for Transnacional Education (GATE, por su sigla en inglés) identifica seis modalidades de educación superior allende las fronteras de los países con sus respectivos ejemplos: (1) Educación a distancia on line: Atlantic International University, Honolulu; (2) Educación a distancia apoyada localmente; Instituto Tecnológico de Monterrey, con apoyo local en varios países de Centroamérica; (3) Programas gemelos (twins): impartidos localmente con el mismo currículo y materiales de estudios utilizados por la universidad extranjera, Universidad del Valle, Guatemala, y una línea similar Universidad Nacional Pedro Henríquez Ureña (UNPHU), República Dominicana; (4) Programas articulados: el estudiantado cursa una parte de la carrera en una universidad local y el resto en la universidad extranjera, varias universidades colombianas; (5) Sedes locales de instituciones extranjeras: Florida State University (FIU, por su sigla en inglés) o Nova Southeastern University, con sedes en Panamá; y (6) Acuerdos de franquicia: permiso o licencia otorgado a una universidad por otra, forma menos explorada en América Latina y el Caribe. Otra forma no contemplada en esas modalidades, es la alianza estratégica; Instituto Centroamericano de Administración de Empresas (INCAE) con la Escuela de Negocios de la Universidad de Harvard.

Esta misma autora advierte que los nuevos desarrollos de la internacionalización de la educación superior pueden acarrear consecuencias no intencionadas, como la competencia entre países para atraer personas brillantes y con más talentos, a estudiar y trabajar en sus instituciones ("ganancia" de cerebros) o, en los últimos tiempos, el surgimiento del término "entrenamiento" de cerebros (interés cada vez más frecuente de estudiantes y de personal investigador por obtener un segundo grado o pasantía en una universidad de otro país). Esas nuevas oportunidades (éxitos y también amenazas) exigen alerta y resulta imperativo que la internacionalización de la educación superior siga siendo proactiva, responsable e innovadora, pues a medida que madura un ojo crítico, una fuerte voluntad resulta necesaria para darle seguimiento a sus resultados, implicaciones y consecuencias (intencionadas y no intencionadas) (Knight, 2010).

De acuerdo con la misma Knight (1994), en términos generales, es posible distinguir cuatro estadios de desarrollo de la internalización de la educación superior: (1) Institucional: integración de elementos y perspectivas internacionales en el ejercicio de las funciones sustantivas. (2) Programática: desarrollo curricular, intercambio docente y estudiantil y cooperación técnica. (3) Desarrollo de competencias internas: en estudiantes, académicos y administrativos. (4) Estructura organizacional: ethos o cultura que valore y apoye las perspectivas e iniciativas internacionales e interculturales. Sin lugar a dudas, la etapa más díficil. 
2004); oportunidades y desafíos (Aupetit, 2007); integración regional (Miranda et al., 2008); conceptualización en la perspectiva de otros enfoques y términos empleados, y el significado de su definición operativa (Jaramillo, 2009), referentes para su evaluación (Ceja Mendoza, 2010) y, en forma más específica, balance de proveedores externos (Estrada Muy y Luna, 2004), hasta la doble titulación (Universidad Nacional de Colombia, 2007).

El otorgamiento de títulos allende las fronteras de los países cobra interés como modalidad de alto impacto en el posicionamiento y escalamiento de estadios superiores en la internacionalización de la educación superior. En América Latina y el Caribe empieza a desarrollarse en forma creciente $y$, por su intermedio, el estudiantado tiene acceso a un título otorgado en forma simultánea por dos instituciones de educación superior (incluso más), suscrito en forma conjunta o bien uno de cada universidad ${ }^{7}$.

Un estudio realizado en la Universidad Nacional de Colombia sistematiza su importancia como sigue:

Lo más importante de la doble titulación es lo que hay detrás de ella, puesto que es muy importante el esfuerzo mancomunado entre dos instituciones que tratan de ponerse de acuerdo. Este esfuerzo implica programar, producir seminarios e investigar en conjunto, especialmente en los posgrados. Es ofrecer un sistema de movilidad de los estudiantes y los profesores, que sea coherente y realista. Que no responda simplemente a la espontaneidad, gestos o iniciativas de un profesor, sino a la iniciativa de dos programas respaldados institucionalmente. Universidad Nacional de Colombia (2007, p. 3).

Resumiendo, entre los principales beneficios de la doble titulación internacional, se destacan: (1) la interculturalidad, sin descontextualizar al estudiantado de su medio, y se obliga al profesorado a realizar mayores esfuerzos de aplicabilidad a distintas realidades; (2) posibilidad de tener acceso a un título reconocido en el exterior y con mayor prestigio en el ámbito nacional, sin necesidad de abandonar su lugar de origen y su trabajo; y (3) fomento de la excelencia académica, pues las universidades saben que para mantener su prestigio internacional deben ser exigentes en los controles de calidad.

Otros beneficios adicionales se ubican en la transferencia de conocimiento, el mejoramiento de condiciones para su apropiación, la creación de oportunidades para el desarrollo de

6 Colombia es, quizás, el país que más destaca en el debate actual sobre la internacionalización de la educación superior. A principios de 2000 ya contaba con la Red Colombiana para tal fin, desde 2008 trabaja en conjunto con el Ministerio de Educación en acompañamiento a entidades de educación superior; además, periódicamente realiza encuentros y foros sobre el tema. En Chile, los servicios de enseñanza de la educación superior forman parte de la oferta exportable de la Dirección de Promoción de las Exportaciones (ProChile).

7 En otro nivel, doble titulación también se utiliza para designar la opción que brindan algunas universidades, generalmente a estudiantes con distinción académica, de cursar dos programas al mismo tiempo o dos especialidades en el caso de posgrado, y tener acceso a dos títulos a la vez. Por regla general, la institución ofrece arreglos de horarios y otras facilidades, incluidos descuentos en el pago de matrícula y otros beneficios académicos y económicos adicionales. 
investigaciones y publicaciones conjuntas; así como la inserción temprana de estudiantes en comunidades científicas; e, incluso, un mejor posicionamiento en el acceso a recursos nacionales e internacionales.

\section{Propósito y metodología}

En este artículo se sistematiza la experiencia de una maestría en doble titulación de la Universidad Nacional (UNA) en Costa Rica. Se trata de un programa de formación del Centro Internacional de Política Económica para el Desarrollo Sostenible (CINPE), instancia de investigación que también desarrolla actividades docentes en el nivel de posgrado, impartido en forma conjunta con el Instituto Tecnológico de Santo Domingo (INTEC) de la República Dominicana, hasta el momento, la primera iniciativa en su género conocida en el país y, quizás, de Centroamérica, donde una universidad costarricense aporta el currículo y asume el liderazgo académico en la conducción de un programa de esa naturaleza ${ }^{8}$.

El objetivo fundamental de este artículo es difundir lecciones útiles de la experiencia, a la luz de los crecientes desarrollos de la teoría y práctica de la internacionalización de la educación superior, que puedan ser retomadas con propósitos diversos para otras iniciativas similares.

La sistematización se fundamenta en resultados de distintas instancias de evaluación (autoevaluación institucional, evaluación participativa de estudiantes y docentes y taller de evaluación con representantes de instancias paraacadémicas). La presentación de los principales hallazgos se estructura con base en cuatro ejes de análisis relevantes para los propósitos buscados, a saber: (1) el contexto, (2) la creación de condiciones iniciales, (3) la gestión y gerencia y (4) los factores intervinientes en la ejecución.

\section{El contexto}

La Maestría en Gerencia del Comercio Internacional (MGCI) en doble titulación, cuya experiencia es objeto de la presente reflexión, se trata de un programa inicialmente diseñado e instaurado en el CINPE en 1999, en el marco de un proceso de ampliación y diversificación de la actividad docente, la de más larga tradición en el instituto, pues este surge justamente a partir de la experiencia y trayectoria de la Maestría en Política Económica para Centroamérica y el Caribe, fundada en 1985, mediante un Convenio de Cooperación Académica entre la Universidad Católica de Brabant, en Tilburg, Holanda y la UNA con el apoyo financiero del Gobierno de

8 De hecho, el rector actual y la rectora anterior de la UNA han sido los primeros incumbentes en juramentar graduandos y graduandas en esa modalidad. De acuerdo con una consulta realizada al Consejo Nacional de Rectores (CONARE), ente regulador de la educación superior en Costa Rica, no existe antecedente de un programa de tal naturaleza. En el mismo sentido se pronunció el Consejo Nacional de Enseñanza Superior Privada (CONESUP), entidad que indicó que en Costa Rica ninguna institución de educación superior privada cuenta con autorización para desarrollar programas de doble titulación. 
los Países Bajos. De modo que la vocación internacional resulta consustancial al origen de la institución, como se manifiesta incluso, en sus formas de denominación?.

A lo largo de su trayectoria, el CINPE capitaliza y potencia su vocación inicial, consolidando el acercamiento con universidades europeas, captando oportunidades de formación doctoral para su personal académico e investigaciones conjuntas. Además, desarrolla capacidades institucionales y expertise profesional en diversas fases de gestión, negociación, formulación, ejecución, administración y evaluación de iniciativas académicas de alta complejidad. Animado por su relación académica inicial en el marco de cooperación norte-sur, se va posicionando como par internacional de universidades escandinavas y de un consorcio de universidades holandesas, empezando a actuar como puente de cooperación norte-sur en proyectos conjuntos en Centroamérica (Guatemala es un ejemplo) y fuera del continente (Benín y Bután), lo que le permite acumular liderazgo académico para la cooperación sur-sur con la República Dominicana.

En síntesis, derivado de la experiencia pareciera útil cumplir primero con un ciclo de internacionalización de la educación superior en casa; base para asumir, posteriormente, el desafío del liderazgo hacia fuera y entender mejor la transculturalidad como parte de ese proceso.

\section{La creación de condiciones iniciales}

De la experiencia sistematizada se deriva también que la creación de condiciones iniciales implica, por un lado, el desarrollo sostenible de un ambiente de confianza y empatía entre las entidades académicas participantes, producto de un acercamiento y conocimiento mutuos $y$, por el otro, la conducción de un acertado proceso de toma de decisiones, que permita transitar del requisito de viabilidad inicial al de viabilidad resultante.

En el caso de la MGCI del CINPE-UNA, la doble titulación con el INTEC surge en un contexto en el que ya existía vinculación entre ambas entidades, incluso un convenio de cooperación suscrito y adelantada movilidad académica ${ }^{10}$. En ese marco se gesta la propuesta para impartir la Maestría en forma conjunta ${ }^{11}$, en un área temática que responde a una necesidad de formación a nivel

9 De hecho, desde la primera promoción de la Maestría participan estudiantes de Centro América y de República Dominicana y luego de Jamaica, Haití y Barbados; asimismo como parte de su planta académica se integró profesorado holandés y de distintas nacionalidades europeas, latinoamericanas y norteamericanas. Resulta interesante mencionar que es hasta épocas más recientes cuando las autoridades de la UNA incorporan la internacionalización de la educación superior en su discurso. En efecto, es en el Plan Estratégico Institucional 2007-2011, cuando, por primera vez en la historia institucional, los objetivos estratégicos incluyen como referente académico un posicionamiento en los ámbitos nacional e internacional.

10 El primero de la UNA con una entidad de educación superior en la República Dominicana, cuya firma fue favorecida por los vínculos previos de la entonces directora general del CINPE y viene a cristalizar una aspiración presente desde la creación de la Maestría de Política Económica, el acercamiento con el Caribe. Las características económicas, sociales, culturales e institucionales de la República Dominicana, incluida la cercanía geográfica y las regulaciones para las instituciones de educación superior, incidieron en la articulación de una trayectoria de vinculación (intercambio docente y publicaciones, entre otras actividades) con prestigiosas entidades académicas en ese país.

11 La visión provino del rector del INTEC en ese momento, quien conocía de la Maestría de Política Económica desde la década de 1990 y se interesó en estrechar vínculos académicos al escuchar de su transformación en CINPE. 
de maestría y en un ámbito en el cual la entidad huésped ya había incursionado con otro socio académico internacional. Así, el INTEC, conocido y reconocido por su trayectoria de excelencia académica, su naturaleza jurídica (asociación sin fines de lucro), su grado de institucionalidad y su trayectoria de internacionalización y experiencia de articulación con universidades extranjeras $^{12}$, se decanta como aliado natural para el programa de doble titulación ${ }^{13}$.

Esta experiencia también ilumina que la toma de decisiones para instaurar un programa de doble titulación debe considerar al menos tres ámbitos fundamentales de intervención: (1) análisis de viabilidad, la valoración positiva reafirmaba una expectativa que el programa docente del CINPE se había planteado tiempo atrás: Ilevar la formación al lugar de origen y residencia del estudiantado, en lugar de atraerlo a Costa Rica, como una alternativa para subsanar las implicaciones de costo económico y desarraigo cultural y familiar que profesionales insertos en el mercado laboral no están dispuestos a asumir; (2) estrategia de comunicación, diseñada e instrumentada por medio de visitas a responsables de instancias de toma de decisión, potenciada con la apertura de un canal de comunicación directo por vía electrónica, respaldado con el envío de notas formales, hacia lo interno, con las autoridades y mandos medios de la UNA, y con el propio INTEC ${ }^{14}$; y (3) ruta-crítica, la principal lección aprendida es que la adaptación del plan de estudios significa un amplio proceso de análisis y consulta, tendiente a: (1) conciliar el contenido de cada uno de los cursos con la realidad nacional, (2) armonizar el currículo con la normativa vigente en la materia en ambos países y (3) formular el plan de estudios en el formato exigido para su aprobación por el ente regulador del país huésped.

Resumiendo, la creación de condiciones iniciales para impartir una maestría en doble titulación, consiste en una trayectoria ardua, complicada y de largo alcance, cuya maduración requiere tiempo y no ocurre de la noche a la mañana.

\section{La gestión y gerencia}

La experiencia sistematizada indica que una maestría de doble titulación implica definir, previamente y en forma transparente, el manejo institucional y financiero. En este caso se

12 Su experiencia de asociación para impartir maestrías en doble titulación es amplia y variada e incluye universidades europeas.

13 En palabras del director general de aquel entonces, los criterios utilizados para evaluar la importancia y alcances de la propuesta y tomar la decisión de acogerla e impulsarla, fueron: (1) La oportunidad para dar un paso en un sentido nuevo, y (2) La existencia de antecedentes académicos muy variados. En el caso del país de destino, se conjugaban varias precondiciones a favor: a) Nexos previos con universidades de ese país. b) Procesos de intercambio y cooperación con el INTEC, que garantizaba conocimiento mutuo previo. c) Un grupo de graduados y graduadas de la Maestría de Política Económica, con destacado desempeño profesional en el área, base para una ampliación de la oferta académica del CINPE.

14 Dos acciones ocuparon la atención de manera especial en este tramo de actuación: (1) el acercamiento y conocimiento del funcionamiento de un programa de doble titulación nacional impartido en la UNA; y (2) la determinación de la inexistencia de incompatibilidades con la normativa vigente, tanto en el interior de las universidades como en la reglamentación de los entes reguladores. 
definieron las instancias de toma de decisiones ${ }^{15}$. La coordinación se asume en forma colegiada y con apoyo de asistencia académica. La selección del cuerpo docente se realiza en forma consensuada y atendiendo estrictos estándares de calidad académica: (1) congruencia del perfil profesional, nivel académico y experiencia profesional con el curso por impartir y (2) calidad humana: tolerancia cultural y don de gente. La designación puede que requiera el aval de instancias colegiadas; no obstante, los nombramientos son tramitados por cada entidad de acuerdo con las políticas y procedimientos administrativos internos.

Las actividades de difusión, motivación y extracurriculares resultan de particular relevancia y ocupan un lugar privilegiado en los medios de comunicación de ambas entidades. Mención aparte merecen los encuentros de inserción-inmersión, con un viaje virtual por las instalaciones del CINPE como recurso y el viaje de estudios con propósitos académicos y de intercambio cultural, ampliamente valorado, que incluye lecciones conjuntas en el CINPE y visitas institucionales. Un efecto demostrativo no previsto es que estudiantes del programa regular del CINPE también están realizando viajes de estudio al INTEC.

Desde el inicio, para el INTEC estuvo claro el liderazgo académico del CINPE en virtud de su aporte de: (1) el programa curricular y (2) un cuerpo docente con formación y experiencias internacionales $y$, por ende, el ejercicio de la titularidad de los derechos de producción intelectual, la coordinación general y los parámetros para el establecimiento de la auditoría de calidad. Mientras que, para el CINPE, también resulta claro que esta experiencia innovadora, orientada a la configuración de un espacio académico basado en una comunidad de intereses compartidos, solamente tendría cabida en el marco de una relación horizontal, de cooperación, confraternidad y solidaridad, pues el principio básico de la cooperación es el trabajo conjunto para un mismo fin.

En pocas palabras, la gestión y gerencia de una maestría de doble titulación implica acudir en forma permanente y constante a soluciones innovadoras y consensuadas.

\section{Los factores intervinientes en la ejecución}

Desde el punto de vista académico e institucional, la experiencia de la MGCI del CINPE-UNA en doble titulación con el INTEC devela procesos de trabajo -al menos 10-, cuyos ámbitos de acción rebasan las competencias del programa y que inciden directamente en la ejecución: (1) Calendarización: las fechas de inicio y finalización de los ciclos lectivos no resultan coincidentes necesariamente; y, por ende, existe desfase en los correspondientes trámites académicos (empadronamiento, matrícula, reporte de calificaciones y otros). (2) Asignación de códigos a los cursos: que deben ser diferentes a los correspondientes al programa de titulación nacional, dado que la doble titulación debe responder a un plan de estudios propio. (3) Empadronamiento:

15 Las instancias de auditoría de la calidad académica son las siguientes: (1) Comité de Admisiones y (2) Comité de Gestión Académica. 
sujeto a diferente fechas, formularios, requisitos y documentación, lo cual eleva las exigencias del programa y genera confusión, lo ideal sería que fuera suficiente que se realice únicamente en la entidad sede del programa y sin necesidad de solicitar información superflua y documentación que no aporte valor agregado. (4) Matrícula: al igual que el trámite anterior, lo ideal sería que fuera suficiente con que realice únicamente en la entidad sede y no en las dos entidades, y que los sistemas cuenten con una interfase de comunicación. (5) Presentación título de grado: si el empadronamiento y la matrícula se centralizan en la entidad sede, automáticamente se elimina la complicación, más en este caso en que en el país sede los controles de fidelidad están mejor establecidos ${ }^{16}$. (6) Reporte de calificaciones: debe liberarse de las subjecciones aplicadas a los programas nacionales, o bien centralizarse en las coodinaciones. (7) Firma de actas de calificaciones: debe estar sujeta a los mismos criterios que el reporte de calificaciones. (8) Plataformas tecnológicas: su diseño y tipo de acceso deben concordar con criterios y parámetros de internacionalización. (9) Evaluación: debe asumirse en forma consensuada una única hoja de evaluación, también sujeta a criterios y parámetros de internacionalización. (10) Graduación, juramentación y entrega de títulos: la calendarización y requisitos administrativos deberían flexibilizarse y generar un protocolo aplicable únicamente a la emisión de títulos de programas en doble titulación.

En resumen, una advertencia de alto calibre es que hace falta poner la debida atención en la armonización de procedimientos, instrumentos y formularios; $y$, por regla natural, debería prevalecer el calendario y los procedimientos administrativos instaurados en la entidad húesped.

\section{Conclusiones}

Una primera conclusión general, pero relevante, si bien la internacionalización de la educación superior es consustancial al origen mismo de la universidad, dado su carácter de universal, es posible afirmar que la novedad en términos recientes radica en la frecuencia y diversidad de modalidades, cuyo flujo aparece ligado a la creciente y compleja interdependencia entre países y al acortamiento de la distancia (en forma presencial y virtual), dado el vertiginoso e inminente avance de las tecnologías de información y comunicación.

Entre las distintas posiciones de la internacionalización de la educación superior destaca la que la concibe como integración de las dimensiones internacional e intercultural, pues permite avanzar hacia un modelo novedoso de trabajo entre entidades académicas que puede trascender la cooperación entre países.

Otra conclusión general, pero también significativa, es que para incursionar de manera exitosa en la internacionalización de la educación superior hace falta reunión de voluntades,

16 Mención aparte merece este proceso, pues implica una considerable erogación de recursos y tiempo. La suscripción de Costa Rica de La Apostilla de La Haya ha logrado simplificar un paso y un pago. 
tiempo, esfuerzo, recursos; constancia y compromiso de las partes interactuantes, más allá de las responsabilidades institucionales; visión, compromiso institucional, voluntad política, perseverancia académica, métodos de trabajo basados en procesos de planificación y evaluación estratégica, que trasciendan la cotidianidad y relancen la proyección internacional más allá de las simples rutinas de trabajo.

La principal lección aprendida con la MGCI del CINPE-UNA de doble titulación con el INTEC es que la instauración de un programa de esa naturaleza, de ninguna manera implica la repetición automática y acrítica de un plan de estudios formulado, aunque este se haya puesto en práctica en numerosas ocasiones o haya sido evaluado.

Entre los factores clave determinantes de los logros obtenidos se destacan: (1) las relaciones de respeto y confianza establecidas entre las coordinadoras; (2) la comunicación permanente en forma presencial y electrónica, y el hecho de que ambas cuentan con un canal de comunicación directo con las instancias superiores, especialmente con la rectoría de ambas universidades; (3) un apoyo calificado por parte de las asistentes académicas y el personal secretarial, que les permite dedicar tiempo de calidad a la selección del cuerpo docente y al seguimiento académico, (4) los criterios utilizados en la selección docente, (5) el método de inserción-inmersión, que incluye el briefing y debriefing del profesorado. En un lugar especial hay que mencionar que tanto el profesorado como el estudiantado manifiestan identificación, compromiso y motivación para cumplir con las exigencias académicas del programa y, sobretodo, destaca el marcado carácter de transculturalidad como valor agregado del proceso de formación.

Una mirada en conjunto de esta iniciativa muestra pertinencia y coherencia de los propósitos buscados, que se manifiesta en: (1) el estudiando se muestra satisfecho con el programa, ya que responde a sus expectativas y a las necesidades educativas nacionales en el nivel de maestría. (2) En su organización, el programa favorece un ambiente adecuado de aprendizaje y facilita a sus estudiantes el acceso a conocimientos, información y experiencias útiles para su enriquecimiento profesional. (3) En la toma de decisiones, se dispone de instancias articuladas y coordinadas de mecanismos de comunicación eficientes, y de una cultura de planificación y evaluación estratégica. (4) Por medio del programa, tanto el INTEC como el CINPE han visto realizada su aspiración de ampliar su oferta académica de programas internacionales.

Un balance de la relación entre las dos entidades académicas evidencia que la asociación las ha beneficiado. El equilibrio de las responsabilidades ha sido adecuado, y sobresalen el apoyo y las relaciones de confianza entre todas las partes interesadas. El modelo de consorcio académico, basado en relaciones de cooperación y complementariedad, en igualdad de condiciones y en forma horizontal, con roles académicos establecidos, delimitados y diferenciados, ha demostrado eficacia. En la práctica se está construyendo un modelo de trabajo asociado con una práctica de gestión universitaria, cuyos resultados merecen amplia difusión. 
La eficacia del modelo de trabajo se ve disminuida ante la necesidad de alcanzar una fórmula de eficiencia más razonable, dado que está ligada a los procedimientos internos de las instituciones y que, en el caso de la UNA, aún está pendiente la agenda de renovación institucional con el propósito de asumir la internacionalización en todas sus dimensiones, por lo que queda fuera del ámbito de acción de la matriz de la ejecutoria. Las autoridades institucionales deberían ser capaces de emitir señales claras que posibiliten una real simplificación de procesos internos en aras de una gestión más ágil y expedita, y los mandos medios, a la vez, deberían velar porque las contradicciones internas no se traduzcan en innecesarios y evitables costos de transacción. Esto permitiría que, en la práctica, un programa de doble titulación no se convierta en una "doble complicación" para las distintas partes involucradas, con el consecuente desgaste de tiempo y recursos que ello significa.

La sostenibilidad de la iniciativa exige la resolución de las dificultades que atentan contra la eficiencia del modelo de trabajo, pues un costo de transacción relativamente alto terminará agotando a quienes tienen a cargo la coordinación. Las exigencias crecientes y permanentes de información y documentación conducirán a la búsqueda de socios académicos que ofrezcan mayores márgenes de flexibilidad y grados menores de complejidad, o habrá que conformarse con operar en situaciones menos complicadas y demandantes.

En cuanto al impacto, es claro que se observan mejoras cualitativas en distintos ámbitos de acción: (1) en el estudiantado: razón de ser de las instituciones educativas que consideran que están ampliando su acervo de conocimientos y experiencias prácticas por medio del proceso de formación que les brinda la Maestría. Además, los aspectos académicos del programa (cuerpo docente y plan de estudios) juegan un papel clave en su crecimiento personal y profesional, sobre todo, los rasgos asociados a la internacionalización (contenidos de los programas, expertise del profesorado, viajes de estudios, etc.); (2) en el cuerpo docente, que dispone de un espacio para ampliar su horizonte de transculturalidad y de enriquecimiento profesional, gracias al contacto con una realidad nacional distinta de la propia, aunque con similitudes que le permiten entender otros contextos y realizar una relectura de la realidad internacional; (3) en las instancias académicas involucradas que en la práctica están construyendo y validando a partir del aprendizaje por experiencia, a menudo casi sin proponérselo, un modelo de relacionamiento académico pertinente y eficaz que puede constituirse en una buena práctica para la internacionalización de la educación superior mediante la cooperación sur-sur.

La institucionalización de una experiencia no significa continuar repitiéndola siempre de la misma manera; sino, más bien, lograr la legitimidad social de los procesos para que adquieran identidad y vida propia. Sin lugar a dudas, la ejecución es el ámbito que requiere más mejoras. En la práctica, en el caso de la UNA, se han flexibilizado fechas, apertura y extensión de plazos, pero no se han readecuado y normalizado las condiciones requeridas por las demandas de un programa de doble titulación. 
Finalmente, la experiencia muestra que el respeto a la voz de la academia en la construcción de la lógica de las intervenciones garantiza la calidad del diseño, el involucramiento y compromiso de toda la comunidad académica; situación que, además, repercute de manera directa en las fases de gestión, negociación, ejecución, administración, seguimiento y evaluación.

\section{Referencias}

Ceja Mendoza, A. (2010). Una aproximación a la política de internacionalización de la educación superior. Referentes para su evaluación. Revista Educación y Desarrollo 14, Julio - Setiembre.

Chadee, D. y Naidoo, V. (2009). 'Higher Education Services Exports: Sources of Growth of Asian Students in US and UK', Service Business, vol.3:2, p. 173-87.

Estrada Muy, M. y Luna, J. G. (2004). Internacionalización de la educación superior. Nuevos proveedores externos en Centroamérica. UNESCO y CSUCA. Informe de Avance. Guatemala. Disponible en http://www.inca-network.org/public documents/Internacionalizacion ES CA.pdf

Gacel, J. y Ávila, R. (2008). Universidades latinoamericanas frente al reto de la internacionalización. Revista Casa del Tiempo, No.9, Julio.

Gacel-Ávila, J. (2003) Internacionalización de la educación superior: Paradigma para la ciudadanía global. México: Centro Universitario de Ciencias Sociales. Universidad de Guadalajara.

Gacel-Ávila, J. (2005). La internacionalización de la educación superior en América Latina: El caso de México. Cuaderno de Investigación en la Educación 20. México: Universidad de Guadalajara.

Gate. (s. f.).The Gate Certification Process. Disponible en http://www.edugates.org.

Gazzola, A. L (2008): Integración académica e internacionalización de la educación superior. Disponible en http://www.cuib.org/documentos/documentos/ALGazzola.pdf

Gazzola, A. L. y Didriksson, A. (editores) (2008). Tendencias de la educación superior en América Latina y el Caribe. IESALC: Caracas: IESALC (Instituto Internacional de la UNESCO para la Educación Superior en América Latina y el Caribe).

Knight, J. (1994). Internationalization: Elements and Checkpoints. CBIE Research 7. Canadian Bureau for International Education.

Knight, J. (2003). GATS, Trade and Higher Education-Perspective 2003-Where Are We? Policy paper published by the Observatory on Borderless Higher Education, London, UK. 
Knight, J. (2004). Internationalization Remodeled: Definitions, Approaches and Rationales. En Journal of Studies in International Education, 8. Disponible en: http://jsi.sagepub.com/ content/8/1/5.short

Knight, J. (2007). Internationalization: Concepts, Complexities and Challenges. Springer International Handbooks Education, Volume 18 of the series p. 207-227.

Knight, J. (2010). Internacionalización de la educación superior: Nuevos desarrollos y consecuencias no intencionadas. Boletin IESALC Informa de Educación Superior, 211.

Ramírez Sánchez, C. (2004). La internacionalización de la educación superior en Chile. Disponible en: http://www.ita-chile.cl/prontus ita/site/artic/20060915/asocfile/20060915134640/sobre la internacionalizacion.pdf

Universidad Nacional (2007): Plan estratégico institucional 2007-2001. Heredia, Costa Rica: Autor.

Universidad Nacional de Colombia (noviembre, 2007): Doble titulación. Un camino a la internacionalización. Claves para el debate público, 8. Unimedios. Bogotá, Colombia:Centro de Información. Disponible en http://www.agenciadenoticias.unal.edu.co/uploads/media/Claves 8 02.pdf 